\title{
Portobiliary fistula: unusual complication of wire- guided cannulation during endoscopic retrograde cholangiopancreatography
}

Wire-guided cannulation (WGC) is widely used for bile duct cannulation during endoscopic retrograde cholangiopancreatography (ERCP) [1]. Here we present a patient with a WGC-induced portobiliary fistula.

A 65-year-old man was admitted to our department for further evaluation of a dilated segment IV bile duct detected on computed tomography (CT). There was no evidence of dilated veins or aberrant vessel development around the pancreatic head on CT. The physical examination and laboratory tests were unremarkable. Duodenoscopy revealed no masses in the ampulla of Vater ( Fig. 1 a). To cannulate the bile duct for further evaluation, WGC using a conventional catheter was attempted. A hard 0.025-inch guide wire was gently and carefully advanced under endoscopic and fluoroscopic guidance ( Fig. 1b). After three attempts, we assumed that selective bile duct cannulation was achieved because the guide wire advanced smoothly ( $\bullet$ video 1 ).

The catheter was then inserted over the guide wire with slight resistance near the ampulla of Vater. On cannulation, when we could not aspirate bile or blood, contrast medium was injected through the catheter, which cleared within a few seconds in the direction of the liver ( Fig. 2). We also recognized subtle blood flow, which we speculated as arising from the posterior superior pancreatoduodenal vein around the pancreatic head $(\bullet$ Video 2 ).

We realized that the catheter was placed in the portal venous system, and the cath-

\section{Video 1}

After three attempts at wire-guided cannulation, we believed that selective bile duct cannulation had been achieved because the guide wire was advancing smoothly.

\section{Video 2}

Radiograph showing subtle blood flow around the pancreatic head.
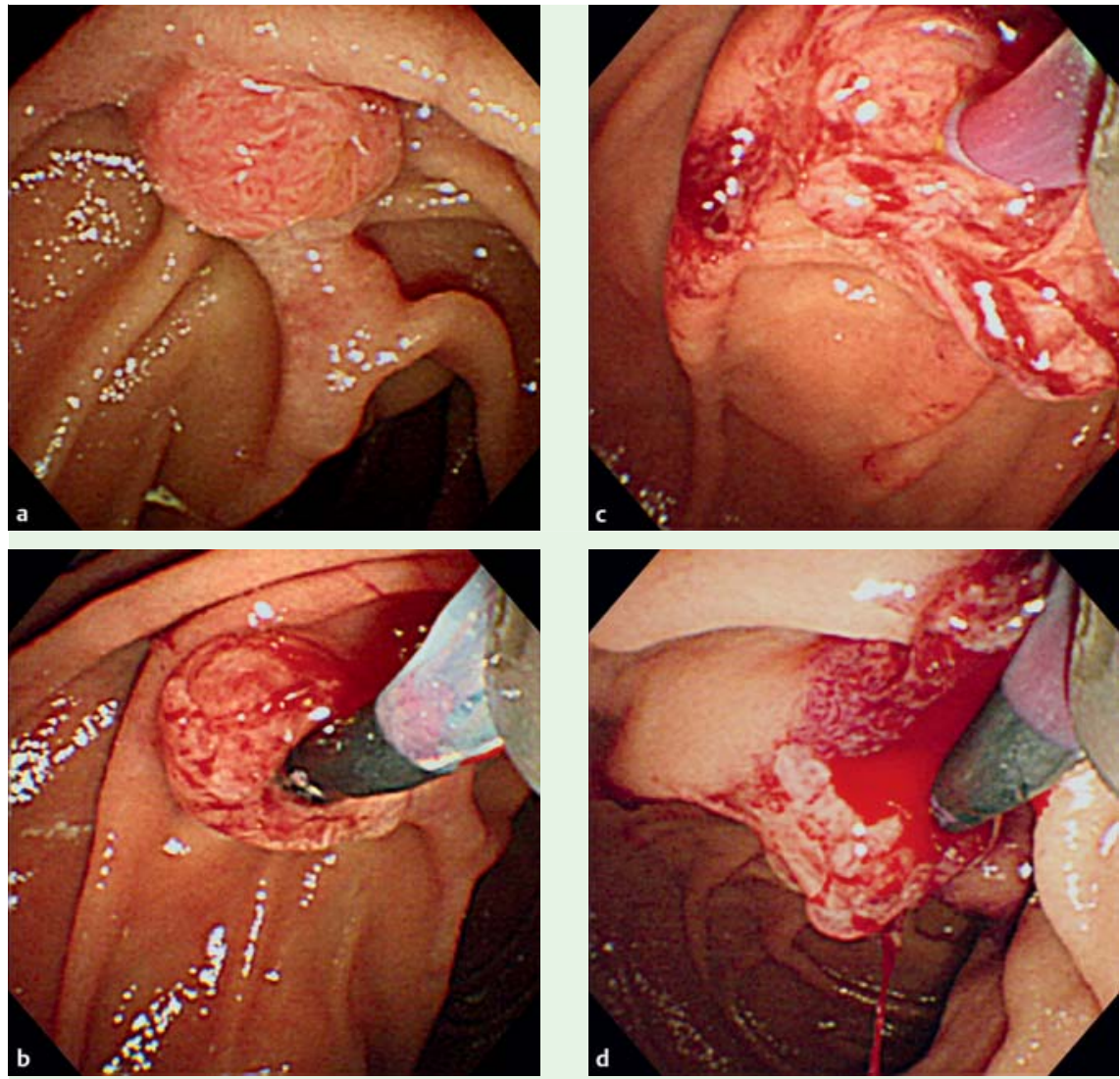

Fig. 1 a Endoscopic image showing no masses in the ampulla of Vater. $\mathbf{b}$ Endoscopic image showing the catheter being gently and carefully advanced over a 0.025 -inch guidewire. c Endoscopic image showing small amounts of blood in the catheter after immediate catheter and guidewire withdrawal from the portal venous system. $\mathbf{d}$ Endoscopic image showing minor bleeding from the ampulla of Vater.

eter and guide wire were immediately withdrawn ( Fig.1c). Duodenoscopy showed small amounts of blood in the catheter and minor bleeding from the ampulla of Vater ( Fig. 1 d).

The patient did not require any treatment for the minor bleeding. Balloon-associated endoscopic retrograde cholangiography (ERC) was carried out 1 week later, but segment IV bile duct was not visualized during this procedure. Cytological analysis of aspirated bile revealed no evidence of malignancy. Based on these radiological and cytological findings, we made the diagnosis of a benign localized biliary stricture as the cause of the dilated segment IV bile duct. We continued care- ful follow-up with laboratory tests and imaging at regular intervals.

Iatrogenic portobiliary fistula is an uncommon complication of biliary drainage, liver biopsy, and surgery, which may result in bleeding, sepsis, portal thrombosis, and air embolism [2]. Cannulation or visualization of the portal vein during ERCP is a rare complication, with an incidence in 1 in $6000-8000$ cases $[3,4]$. This complication during ERCP can result from the laceration of a small portal vein or from direct trauma to the papilla [2]. Serious complications have not been reported. Immediate withdrawal of the tube inserted in the portal vein does not cause serious bleeding [5]. To our knowl- 

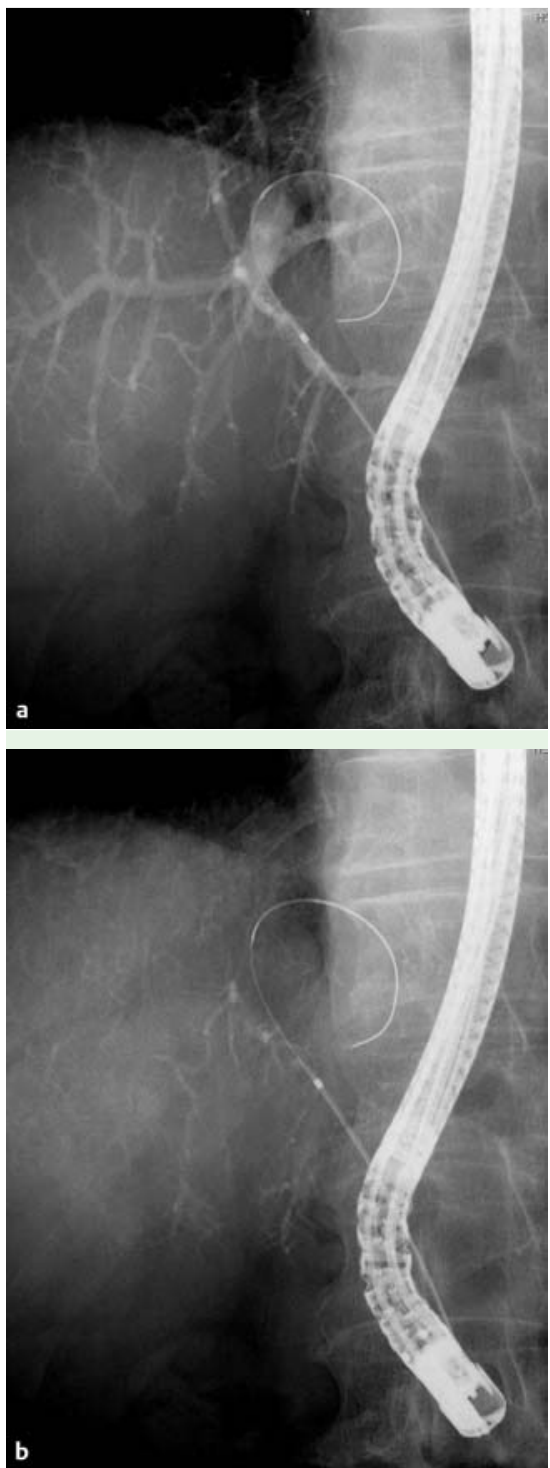

Fig. 2 a Radiograph showing contrast medium injected through the catheter. b Radiograph showing contrast medium cleared within a few seconds without suction. edge, this is the first report of a portobiliary fistula created during WGC using a standard guide wire. More attention should be paid to the possibility of guide wire-related portobiliary fistulas while using the WGC technique.

Endoscopy_UCTN_Code_CPL_1AK_2AD

Competing interests: None

H. Kawakami, M. Kuwatani, T. Kudo, N. Ehira, H. Yamato, M. Asaka

Department of Gastroenterology,

Hokkaido University Graduate School of

Medicine, Sapporo, Japan

\section{References}

1 Bourke MJ, Costamagna G, Freeman ML. Biliary cannulation during endoscopic retrograde cholangiopancreatography: core technique and recent innovations. Endoscopy 2009; 41: 612 -617

2 Espinel J, Pinedo ME, Calleja JL. Portal vein filling: an unusual complication of needleknife sphincterotomy. Endoscopy 2007; 39: E245
3 Ricci E, Mortilla MG, Conigliaro R et al. Portal vein filling: a rare complication associated with ERCP for endoscopic biliary stent placement. Gastrointest Endosc 1992; 38: 524-525

4 Siegel JH, Ben Zvi JS, Yatto RP. Portal vein filling during ERCP. Gastrointest Endosc 1993; 39: $471-472$

5 Furuzono M, Hirata N, Saitou J et al. A rare complication during ERCP and sphincterotomy: placement of an endoscopic nasobiliary drainage tube in the portal vein. Gastrointest Endosc 2009; 70: 588-590

Bibliography

DOI $10.1055 / \mathrm{s}-0030-1256150$

Endoscopy 2011; 43: E98 - E99

(c) Georg Thieme Verlag KG Stuttgart · New York . ISSN 0013-726X

\section{Corresponding author}

Dr. H. Kawakami

Department of Gastroenterology

Hokkaido University Graduate School of Medicine Kita 15, Nishi 7

Kita-ku

Sapporo 060-8638

Japan

Fax: +81-11-706-7867

hiropon@med.hokudai.ac.jp 